\title{
How-I-Do-It
}

\section{Microwave ablation of colorectal liver metastases: Impact of a 10-mm safety margin on local recurrence in a tertiary care hospital}

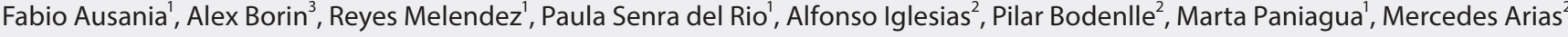 \\ 'Hepatopancreatobiliary Surgery Unit, Department of Digestive Surgery, Vigo University Hospital, Vigo, Spain, \\ ${ }^{2}$ Department of Radiology, Vigo University Hospital, Vigo, Spain, \\ ${ }^{3}$ Liver Transplant Unit, Department of Surgery, Verona University Hospital, Verona, Italy
}

\begin{abstract}
Microwave ablation (MWA) for colorectal liver metastasis (CLM) has been traditionally considered inferior to surgery due to the higher rate of local recurrence. The study investigated whether a safety margin of $10 \mathrm{~mm}$ can improve local control in patients undergoing surgical MWA. Surgical MWA was used to treat 53 lesions in 22 patients with CLM at our Institution from June 2012 to June 2017. The patients' mean age was 64.5 years, and the median size of the lesion was $16.5 \mathrm{~mm}$ (9-34 $\mathrm{mm})$. MWA was associated with liver resection in 16 patients (72.7\%). The median follow-up was 32.4 months. Univariate and multivariate analyses were performed to identify factors associated with tumor recurrence. Median ablation area was $36.6 \mathrm{~mm}^{2}\left(30-50 \mathrm{~mm}^{2}\right)$. The complication rate was $22.7 \%$. No local recurrence was observed during follow-up. Disease-free survival was 20 months (4.8-55.2 months). Univariate analysis revealed that the number of liver metastases and node-positive primary tumors were associated with tumor recurrence. Multivariate analysis revealed that node-positive primary tumor was the only factor significantly associated with tumor recurrence $(p=0.049$; odds ratio, 12 ; $95 \%$ confidence interval, 1-143). When performed with a 10-mm safety margin, surgical MWA can lead to acceptable oncological outcomes with low morbidity. Therefore, it represents a good option in selected patients with CLM.
\end{abstract}

Key Words: Liver; Colorectal neoplasm; Microwaves

\section{INTRODUCTION}

Microwave ablation (MWA) can be used to treat patients with colorectal liver metastasis (CLM) when surgery is not indicated [1]. Although some investigators have reported encouraging oncological outcomes, a local recurrence of 5\%-13\% following MWA for CLM has been reported, suggesting inadequate evidence to recommend MWA as the primary treatment for CLM $[2,3]$.

Received: April 19, 2021, Revised: June 15, 2021,

Accepted: July 11, 2021

Corresponding author: Alex Borin

Liver Transplant Unit, Department of Surgery, Verona University Hospital,

Piazzale Aristide Stefani, 1, Verona 37134, Italy

Tel: +39-45-8123948, Fax: +39-45-8123231, E-mail: alexborin.ab@gmail.com

ORCID: https://orcid.org/0000-0001-8316-2459

opyright (C) The Korean Association of Hepato-Biliary-Pancreatic Surgery

This is an Open Access article distributed under the terms of the Creative Commons Attribution Non-Commercial License (http://creativecommons.org/licenses/by-nc/4.0) which on-commercial use, distribution, and reproduction in any medium, provided the original ermits unrestricted non work is properly cited.
Recent studies suggested that ablation margin is an independent prognostic factor associated with recurrence in patients with hepatocellular carcinoma treated with radiofrequency ablation (RFA). However, there is no consensus on margin extension, and a 5 to $10-\mathrm{mm}$ safety margin is advised, especially in case of larger tumors [4-6].

A recent prospective randomized trial showed significant reduction of tumor recurrence in cirrhotic patients under ChildPugh class B with a single small hepatocellular carcinoma when RFA treatment was used with a targeting margin of 10 $\mathrm{mm}$ [7]. However, none of the studies analyzed the role of 10 $\mathrm{mm}$ safety margin following MWA of CLM.

The aim of our study is to analyze the oncological outcomes of patients with CLM treated via surgical MWA and a $10 \mathrm{~mm}$ safety margin at our Institution. We identified the factors associated with poorer survival in these patients. 


\section{MATERIALS AND METHODS}

We retrospectively reviewed all patients diagnosed with CLM from June 2012 to June 2017 and treated via surgical MWA at our center. Data were extracted from a prospectively maintained database. All demographic, clinical, operative, pathological, and follow-up data were collected.

\section{Inclusion and exclusion criteria}

The inclusion criteria were as follows: liver metastasis based on computed tomography (CT) scan with or without magnetic resonance imaging (MRI) with a histological diagnosis of colorectal carcinoma following biopsy of the primary tumor or a history of colorectal surgery; no previous or simultaneous malignancy; no previous liver surgery or ablation. Only patients indicated for MWA during surgery (open surgical approach) were considered for this study. Open ablation was indicated by a synchronous surgical approach or when laparoscopic ablation was contraindicated. The exclusion criteria were as follows: patients who refused to undergo MWA as the initial ablation treatment; patients with exophytic tumor, perivascular or peribiliary lesions and presence of extrahepatic disease. Perivascular lesions were defined by the location of the nearest margin $\leq 5 \mathrm{~mm}$ from a vessel measuring at least $4 \mathrm{~mm}$ in diameter. Peribiliary lesions were defined as lesions with the nearest margin $\leq 5 \mathrm{~mm}$ from the main right or left hepatic duct. We consider perivascular or peribiliary lesions as a contraindication for MWA at our center. In such cases, we perform irreversible electroporation when feasible. Patients with missing data or patients lost to follow-up (follow-up $<12$ months after ablation) were excluded, as were patients who failed to provide contrast-enhanced CT or MRI results acquired a maximum of 4 weeks prior to the initial procedure. Lesions treated with MWA that were undetectable on pre-procedural CT but found and treated during laparotomy were also excluded from analysis.

\section{Ablation technique}

Intraoperative evaluation of a $10-\mathrm{mm}$ safety margin was performed via intraoperative ultrasonography (US). Once the lesion was reached with an MWA needle via US, the ablation area (circumferentially exceeding the original diameter by 10 $\mathrm{mm}$ ) was defined by entering the appropriate time and wattage. Ablation was monitored via US during the procedure. Further ablations were performed in case of suspected incomplete procedure.

Two experienced radiologists (MA and $\mathrm{AI}$ ) reviewed the imaging data. All patients underwent a contrast-enhanced CT with or without liver MRI 4 weeks after the procedure. Preoperative tumor size and ablation area were compared and a second ablation was considered if necessary. "Local recurrence" was defined by the presence of tumor within, or not dissociated from the ablation area of the MWA zone at follow-up. "Intra- hepatic recurrence" was defined by the presence of tumor in any other liver site. The follow-up imaging protocol involved $3,6,12,18$, and 24 months of follow-up CT scans. Complications were graded according to Clavien-Dindo classification [8]. All procedures were performed according to the guidelines for good clinical practice. Due to the retrospective analysis of these data, a formal review board approval was waived since the patients were not subjected to procedural rules.

\section{Outcome measures}

The primary outcome measure was local recurrence during follow-up. Secondary outcomes were complications and disease-free survival.

\section{Statistical analysis}

Frequencies were compared using chi-squared or Fisher's exact tests. Continuous data were compared using the independent samples t-test or the Mann-Whitney $U$ test, depending on whether or not they were normally distributed. Continuous variables were expressed as medians and/or ranges. Data were previously tested for normality by the Kolmogorov-Smirnov test. The univariate analyses revealed variables associated with disease recurrence. Multivariate analysis of statistically significant variables $(p<0.05)$ was performed using binary logistic regression. Disease-free survival (DFS) was calculated using the Kaplan-Meier analysis. Statistical analyses were performed using IBM SPSS ver. 19 (IBM Corp., Armonk, NY, USA).

\section{RESULTS}

The study analyzed 22 out of 280 patients undergoing liver resection and/or open ablation during the study period. Subjects without CLM (91 patients) and those exposed to surgical resection only (167 patients) were excluded.

Fifty-three lesions involving 22 patients with CLM were consecutively treated with surgical MWA at our Institution from June 2012 to June 2017.

The mean age was 64.5 years, and the median size of the lesion was $16.5 \mathrm{~mm}$ (9-34 $\mathrm{mm})$. Preoperative carcinoembryonic antigen (CEA) level was $7.3 \mathrm{ng} / \mathrm{mL}$. Indications for MWA in CLM patients were: unresectable bilobar metastases with a single-stage procedure in 16 patients $(72.7 \%)$ and high-risk liver resection in 6 patients (27.3\%).

Sixteen patients underwent combined surgical resection and MWA. Fifteen patients underwent parenchyma-sparing minor resection and one patient was treated via major hepatectomy. No R1 liver resections were performed in the 16 patients who received combined MWA and surgical resection. The pattern of these procedures is presented in Fig. 1. Median follow-up was conducted at 32.4 months. Demographic and clinical data are shown in Table 1 . Table 2 presents the different tumor characteristics of patients who underwent surgical resection and MWA compared with patients who underwent only MWA. 

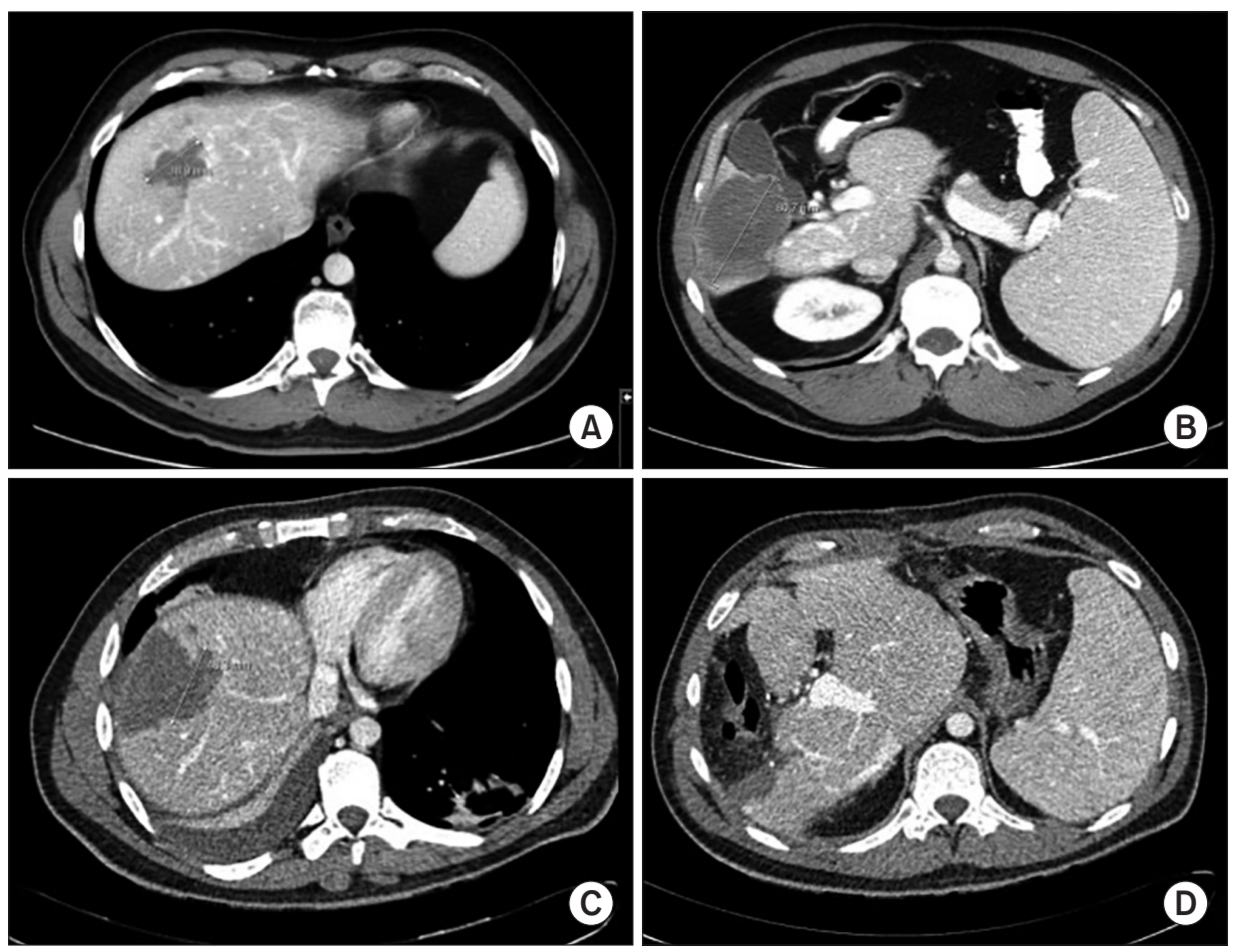

Fig. 1. Computed tomography scan of a patient with multiple liver metastases before the operation $(A, B)$ and 4 weeks after microwave ablation (MWA) and liver resection $(C, D)$. Images $(A, C)$ show liver metastasis before and after MWA with a 10$\mathrm{mm}$ margin. Synchronous liver resection was also performed (B. D).

Table 1. Demographic and clinical data

\begin{tabular}{lc}
\hline \multicolumn{1}{c}{ Variable } & Value \\
\hline Female & $17(77.3)$ \\
Age (yr) & $64.5(40-83)$ \\
ASA score & \\
II & $5(22.7)$ \\
III & $17(77.3)$ \\
Preoperative CEA levels (ng/mL) & $7.3(1.4-26.6)$ \\
Preoperative lesion size (mm) & $16.5(9-34)$ \\
Postoperative ablation area (mm $\left.{ }^{2}\right)$ & $36.6(30-50)$ \\
Patients with synchronous CLM & $17(77.3)$ \\
MWA W & $55.3(20-140)$ \\
Patients with multiple CLM treated with MWA & $19(86.4)$ \\
Wild K-RAS status & $6(27.3)$ \\
Neoadjuvant chemotherapy ${ }^{\mathrm{a})}$ & $12(54.5)$ \\
Adjuvant chemotherapy) & $9(40.9)$ \\
MWA + synchronous liver resection & $16(72.7)$ \\
Postoperative complications & $5(22.7)$ \\
\hline
\end{tabular}

Values are presented as number (\%) or median (range).

ASA, American Society of Anesthesiologists; CEA, carcinoembryonic antigen; CLM, colorectal liver metastasis; MWA W, microwave ablation watts.

a) Liver directed chemotherapy.

Clavien-Dindo 3-4 complications occurred in $22.7 \%$ of patients. No mortality was observed. Median ablation area was $36.6 \mathrm{~mm}^{2}\left(30-50 \mathrm{~mm}^{2}\right)$. One patient received further ablation one month after the first procedure due to suspected residual
Table 2. Tumor characteristics of patients who underwent surgery combined with MWA or only MWA

\begin{tabular}{lccc}
\hline \multicolumn{1}{c}{ Variable } & $\begin{array}{c}\text { Surgical } \\
\text { resection } \\
\text { MWA } \\
(\mathrm{n}=16)\end{array}$ & $\begin{array}{c}\text { MWA only } \\
(\mathrm{n}=6)\end{array}$ & $\begin{array}{c}\text { Total } \\
(\mathrm{n}=22)\end{array}$ \\
\hline Primary tumour location & & & \\
$\quad$ Right-transverse colon & $4(25.0)$ & $3(50.0)$ & $7(31.8)$ \\
Left colon & $8(50.0)$ & $2(33.3)$ & $10(45.5)$ \\
Rectum & $4(25.0)$ & $1(16.7)$ & $5(22.7)$ \\
Synchronous metastasis & $10(62.5)$ & $2(33.3)$ & $12(54.5)$ \\
Bilobar lesions & $14(87.5)$ & $3(50.0)$ & $17(77.3)$ \\
$>$ 3 segment involved & $9(56.3)$ & $1(16.7)$ & $10(45.5)$ \\
RAS status & & & \\
$\quad$ Mutated & $12(75.0)$ & $4(66.7)$ & $16(72.7)$ \\
Wild type & $4(25.0)$ & $2(33.3)$ & $6(27.3)$ \\
Neoadjuvant therapy & $9(56.3)$ & $3(50.0)$ & $12(54.5)$ \\
Preoperative CEA (ng/mL) & $7.3(2.1-23.3)$ & $7.2(1.4-26.6)$ & $7.3(1.4-26.6)$ \\
\hline
\end{tabular}

Values are presented as number (\%) or median (range).

MWA, microwave ablation; CEA, carcinoembryonic antigen.

disease. No local recurrence was detected during follow-up. Median DFS was 20 months (4.8-55.2 months). DFS at 2-year follow-up was $38.0 \%$. Tumour recurrence (systemic or intrahepatic) occurred in 13 out of 22 patients (59.1\%). Liver-only recurrence (not related to primary procedure) was recorded in 8 patients (36.4\%). DFS rates are presented in Fig. 2.

Univariate analysis revealed a number of liver metastases and 


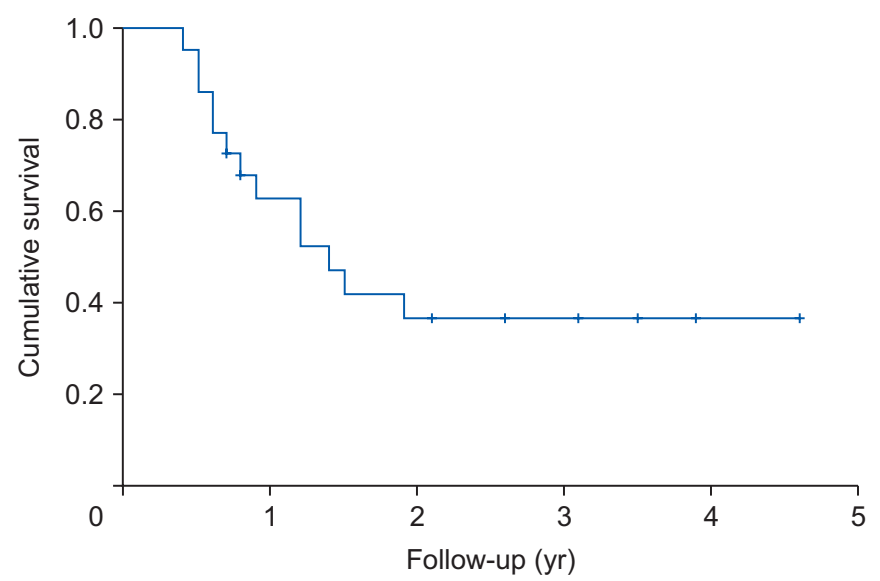

Fig. 2. Disease free-survival (Kaplan-Meier).

node-positive primary tumors associated with tumor recurrence ( $p=0.019$ and 0.013 respectively). Tumor size, synchronous metastasis and CEA > $200 \mathrm{ng} / \mathrm{mL}$ were not associated with tumor recurrence (Table 3).

Results of multivariate analysis revealed that node-positive primary tumor was the only factor significantly associated with tumor recurrence ( $p=0.049$; odds ratio, 12 ; $95 \%$ confidence interval, 1-143) (Table 4).

\section{DISCUSSION}

In this study, we demonstrated that MWA with a $10-\mathrm{mm}$ safety margin was not associated with local recurrence and resulted in low complication rates.

MWA represents a specific form of dielectric heating. Tissue heating occurs as some of the absorbed electromagnetic energy is converted to heat [9]. Surgical resection is still considered the gold standard for the treatment of colorectal liver metastases $[3,10]$. The issue of recurrence has been of great importance, especially in lesions located near large vessels due to the heatsink effect. However, descriptive series comparing survival outcomes in surgical resection and ablation are eclipsed by selection bias [11].

In our study, we did not observe any local recurrence. Postoperative ablation was performed only in one case without compromising the local recurrence rate. The majority of our patients underwent combined surgical and ablation therapy, which could influence the survival analysis. However, DFS in our group of patients was comparable to the reported outcomes following surgical resection and prompted a randomized controlled trial comparing surgical resection with MWA involving 10-mm ablation margin. Beppu et al. [12] described a series of 720 patients undergoing liver resection for CLM and reported DFS rates of $38.5 \%$ and $31.2 \%$ at 2 and 3 years, respectively.

MWA is indicated in two scenarios: bilobar disease not amenable to single-stage resection and high-risk disease that
Table 3. Univariate analysis of factors associated with DFS

\begin{tabular}{lccc}
\hline \multicolumn{1}{c}{ Variable } & $\begin{array}{c}\text { Disease } \\
\text { recurrence } \\
\text { group }\end{array}$ & $\begin{array}{c}\text { No } \\
\text { recurrence } \\
\text { group }\end{array}$ & $p$-value \\
\hline Number of liver metastasis & $4(2-9)$ & $2(1-4)$ & 0.019 \\
Node positive primary tumour & $12(54.5)$ & $1(4.5)$ & 0.013 \\
Tumour size (mm) & $16.2(10-33)$ & $17(9-34)$ & 0.858 \\
Synchronous metastasis & $9(40.9)$ & $4(18.2)$ & 0.662 \\
CEA $>200 \mathrm{ng} / \mathrm{mL}$ & $3(13.6)$ & $1(4.5)$ & 0.474 \\
\hline
\end{tabular}

Values are presented as number (range), number (\%) or median (range). DFS, disease-free survival; CEA, carcinoembryonic antigen.

Table 4. Multivariate analysis of factors associated with DFS

\begin{tabular}{lccc}
\hline \multicolumn{1}{c}{ Variable } & OR & $95 \% \mathrm{Cl}$ & $p$-value \\
\hline No. of liver metastasis & 0.9 & $0.4-7.2$ & 0.892 \\
Node positive primary tumour (n) & 12 & $1-143$ & 0.049 \\
\hline
\end{tabular}

DFS, disease-free survival; OR, odds ratio; $\mathrm{Cl}$, confidence interval.

is contraindicated for percutaneous approach. Most of the patients in our study underwent synchronous surgical resection and the favourable outcomes are in line with other recent studies, which have also demonstrated similar survival rates following single-stage hepatectomy and MWA compared to two-stage hepatectomy, with less overall morbidity [13]. The results of our study open the door to a combined approach for patients with bilobar disease who are candidates for two-stage procedures including preoperative portal vein embolization and associated liver partition and portal vein ligation for staged hepatectomy (ALPPS); long-term outcomes are still limited for these procedures and are associated with high morbidity and mortality [14].

This study has several limitations. First, the number of patients enrolled in this analysis is small. However, the main goal was to assess the local recurrence rate following MWA and therefore 53 procedures may be a fair sample size to study this effect during a nearly 3-year follow-up. Second, all the procedures were performed via open surgical approach which is a more invasive procedure compared with laparoscopy. However, some experts have previously reported that open approach was superior in terms of local efficacy, and laparoscopic ultrasound-guided positioning of ablation needle is a challenge especially for posterior liver segments $[15,16]$. Finally, no control group was available for our analysis, since we started our MWA program in 2012 with a $10 \mathrm{~mm}$ ablation margin, and percutaneous ablations in our centers are performed via RFA. Also, since our local recurrence rate was nil, a comparison group treated with other strategies was not considered.

In conclusion, this is the first study evaluating the impact of a 10-mm MWA ablation margin for the treatment of CLM. In our study, MWA yielded excellent oncological outcomes with 
low morbidity, and therefore represents an acceptable option not only for high-risk patients but also as an alternative to aggressive surgery in selected cases.

However, in the absence of a randomized controlled trial comparing surgical resection with MWA using a $10 \mathrm{~mm}$ ablation margin, thermal ablation should still be reserved for patients who are contraindicated for surgical resection.

\section{CONFLICT OF INTEREST}

No potential conflict of interest relevant to this article was reported.

\section{ORCID}

Fabio Ausania, https://orcid.org/0000-0001-8891-2546

Alex Borin, https://orcid.org/0000-0001-8316-2459

Reyes Melendez, https://orcid.org/0000-0003-1427-4742

Paula Senra del Rio, https://orcid.org/0000-0003-2701-8502

Alfonso Iglesias, https://orcid.org/0000-0003-2371-3786

Pilar Bodenlle, https://orcid.org/0000-0003-0805-6378

Marta Paniagua, https://orcid.org/0000-0002-2951-2582

Mercedes Arias, https://orcid.org/0000-0002-8648-1962

\section{AUTHOR CONTRIBUTIONS}

Conceptualization: All authors. Data curation: FA, AB, AI, MA. Methodology: All authors. Visualization: All authors. Writing - original draft: FA. Writing - review \& editing: All authors.

\section{REFERENCES}

1. Diaz-Nieto R, Fenwick S, Malik H, Poston G. Defining the optimal use of ablation for metastatic colorectal cancer to the liver without high-level evidence. Curr Treat Options Oncol 2017;18:8.

2. Pathak S, Jones R, Tang JM, Parmar C, Fenwick S, Malik H, et al. Ablative therapies for colorectal liver metastases: a systematic review. Colorectal Dis 2011;13:e252-e265.

3. Bala MM, Riemsma RP, Wolff R, Kleijnen J. Microwave coagulation for liver metastases. Cochrane Database Syst Rev 2013;(10):CD010163.

4. Koda M, Tokunaga S, Miyoshi K, Kishina M, Fujise Y, Kato J, et al. Ablative margin states by magnetic resonance imaging with ferucarbotran in radiofrequency ablation for hepatocellular carcinoma can predict local tumor progression. J Gastroenterol 2013;48:1283-1292.
5. Kono M, Inoue T, Kudo M, Chishina $\mathrm{H}$, Arizumi T, Takita M, et al. Radiofrequency ablation for hepatocellular carcinoma measuring 2 $\mathrm{cm}$ or smaller: results and risk factors for local recurrence. Dig Dis 2014;32:670-677.

6. Ke S, Ding XM, Qian XJ, Zhou YM, Cao BX, Gao K, et al. Radiofrequency ablation of hepatocellular carcinoma sized $>3$ and $\leq 5 \mathrm{~cm}$ : is ablative margin of more than $1 \mathrm{~cm}$ justified? World J Gastroenterol 2013;19:7389-7398.

7. Liao M, Zhong X, Zhang J, Liu Y, Zhu Z, Wu H, et al. Radiofrequency ablation using a $10-\mathrm{mm}$ target margin for small hepatocellular carcinoma in patients with liver cirrhosis: a prospective randomized trial. J Surg Oncol 2017;115:971-979.

8. Dindo D, Demartines N, Clavien PA. Classification of surgical complications: a new proposal with evaluation in a cohort of 6336 patients and results of a survey. Ann Surg 2004;240:205-213.

9. Brace CL. Radiofrequency and microwave ablation of the liver, lung, kidney, and bone: what are the differences? Curr Probl Diagn Radiol 2009;38:135-143.

10. Cirocchi R, Trastulli S, Boselli C, Montedori A, Cavaliere D, Parisi $\mathrm{A}$, et al. Radiofrequency ablation in the treatment of liver metastases from colorectal cancer. Cochrane Database Syst Rev 2012;(6):CD006317.

11. Weng M, Zhang Y, Zhou D, Yang Y, Tang Z, Zhao M, et al. Radiofrequency ablation versus resection for colorectal cancer liver metastases: a meta-analysis. PLoS One 2012;7:e45493.

12. Beppu T, Sakamoto Y, Hasegawa K, Honda G, Tanaka K, Kotera Y, et al. A nomogram predicting disease-free survival in patients with colorectal liver metastases treated with hepatic resection: multicenter data collection as a Project Study for Hepatic Surgery of the Japanese Society of Hepato-Biliary-Pancreatic Surgery. J Hepatobiliary Pancreat Sci 2012;19:72-84.

13. Philips P, Groeschl RT, Hanna EM, Swan RZ, Turaga KK, Martinie JB, et al. Single-stage resection and microwave ablation for bilobar colorectal liver metastases. Br J Surg 2016;103:1048-1054.

14. Cai YL, Song PP, Tang W, Cheng NS. An updated systematic review of the evolution of ALPPS and evaluation of its advantages and disadvantages in accordance with current evidence. Medicine (Baltimore) 2016;95:e3941.

15. Eisele RM, Neumann U, Neuhaus P, Schumacher G. Open surgical is superior to percutaneous access for radiofrequency ablation of hepatic metastases. World J Surg 2009;33:804-811.

16. Burdio F, Mulier S, Navarro A, Figueras J, Berjano E, Poves I, et al. Influence of approach on outcome in radiofrequency ablation of liver tumors. Surg Oncol 2008;17:295-299. 